\title{
Iron deficiency in plants: an insight from proteomic approaches
}

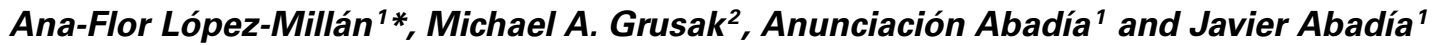 \\ 1 Plant Nutrition Department, Aula Dei Experimental Station (CSIC), Zaragoza, Spain \\ 2 Department of Pediatrics, USDA-ARS Children's Nutrition Research Center, Baylor College of Medicine, Houston, TX, USA
}

\author{
Edited by: \\ Gianpiero Vigani, Università degli \\ Studi di Milano, Italy \\ Reviewed by: \\ Sabine Lüthje, University of \\ Hamburg, Germany \\ Jesus V. Jorrin Novo, University of \\ Cordoba, Spain \\ ${ }^{*}$ Correspondence: \\ Ana-Flor López-Millán, Plant \\ Nutrition Department, Aula Dei \\ Experimental Station (CSIC), \\ Avenida Montañana 1005, E-50059, \\ Zaragoza, Spain \\ e-mail: anaflor@eead.csic.es
}

Iron (Fe) deficiency chlorosis is a major nutritional disorder for crops growing in calcareous soils, and causes decreases in vegetative growth as well as marked yield and quality losses. With the advances in mass spectrometry techniques, a substantial body of knowledge has arisen on the changes in the protein profiles of different plant parts and compartments as a result of Fe deficiency. Changes in the protein profile of thylakoids from several species have been investigated using gel-based two-dimensional electrophoresis approaches, and the same techniques have been used to investigate changes in the root proteome profiles of tomato (Solanum lycopersicum), sugar beet (Beta vulgaris), cucumber (Cucumis sativus), Medicago truncatula and a Prunus rootstock. High throughput proteomic studies have also been published using Fe-deficient Arabidopsis thaliana roots and thylakoids. This review summarizes the major conclusions derived from these "-omic" approaches with respect to metabolic changes occurring with $\mathrm{Fe}$ deficiency, and highlights future research directions in this field. A better understanding of the mechanisms involved in root Fe homeostasis from a holistic point of view may strengthen our ability to enhance Fe-deficiency tolerance responses in plants of agronomic interest.

Keywords: iron, metabolism, proteomics, root, thylakoid

\section{INTRODUCTION}

Iron $(\mathrm{Fe})$ is the sixth most abundant element in the universe and the fourth most abundant in the earth's crust (Morgan and Anders, 1980). For most living organisms on earth, $\mathrm{Fe}$ is an essential element. However, its chemical properties in oxygenated environments limit its availability. As a consequence, Fe deficiency is a widespread phenomenon among the animal and plant kingdoms. According to the WHO, anemia (including Fe-deficiency induced anemia) affects more than 2 billion people worldwide (McLean et al., 2009). In crops, and especially in those grown on calcareous soils, Fe deficiency is a major nutritional disorder that causes decreases in vegetative growth and marked yield and quality losses (Abadía et al., 2011). A substantial body of knowledge has arisen during the genomic era with regard to the molecular aspects of plant $\mathrm{Fe}$ acquisition mechanisms that are induced in response to limited Fe availability (Palmer and Guerinot, 2009; Schmidt and Buckhout, 2011; Hindt and Guerinot, 2012). In all plants except grasses, the adaptation mechanism to Fe limitation suggests the need for increased reducing power (NADH) for the root plasma membrane (PM) Fe reductase, and increased energy (ATP) for the $\mathrm{PM} \mathrm{H}^{+}$-ATPase (Römheld and Marschner, 1983). Proteomic approaches are an excellent tool to elucidate the general metabolic reprogramming needed to sustain these requirements. Understanding how plants respond to Fe limitation at the proteomic level provides a new layer of information on Fe homeostasis processes that can ultimately help to reduce the effects of Fe deficiency in crops. This review summarizes, in a comprehensive manner, the major conclusions regarding metabolic adaptations that occur under Fe deficiency conditions in roots and thylakoids of several plant species, and highlights future research directions in the field. Also, the review provides a clear overview on the limitations of the different proteomic techniques available to study a prevalent abiotic stress case in crops.

\section{CHANGES INDUCED BY FE DEFICIENCY IN THE ROOT PROTEOME}

When Fe is scarce, Strategy I plants develop morphological and biochemical changes leading to an increase in their $\mathrm{Fe}$ acquisition capacity. Morphological changes include swelling of root tips and formation of lateral roots, root hairs, and transfer cells that increase the root's surface area. Biochemical changes result in an increased ability to acquire $\mathrm{Fe}$, and include the induction of a plasma-membrane $\mathrm{Fe}$ (III)-reductase and an $\mathrm{Fe}(\mathrm{II})$ transporter, an enhanced proton extrusion capacity, and the release of low molecular weight compounds such as carboxylates, flavins and phenolic compounds (Abadía et al., 2011).

The most common approach used to study changes in the root proteome upon Fe starvation has been a gel-based approach, using two-dimensional (2-DE) IEF SDS/PAGE electrophoresis. All of the studies reviewed here have been conducted using root extracts of Strategy I plants, whereas proteomic studies in Strategy II plants have only been conducted in root plasma membrane of maize (Hopff et al., 2013). Small gels $(7 \mathrm{~cm})$ were used in studies 
involving Beta vulgaris (Rellán-Álvarez et al., 2010), Medicago truncatula (Rodríguez-Celma et al., 2011a) and Prunus dulcis $\times$ Prunus persica (Rodríguez-Celma et al., 2013a), whereas larger gels were used for Cucumis sativus (24 cm, Donnini et al., 2010) and Solanum lycopersicum ( $24 \mathrm{~cm}$ in Li et al., 2008 and $13 \mathrm{~cm}$ in Brumbarova et al., 2008). When using small gels the average number of proteins detected in root extracts ranged between 140 for B. vulgaris to $\sim 335$ for $P$. dulcis $\times P$. persica and M. truncatula, whereas in larger gels the number of spots ranged from 1400 in S. lycopersicum to 2200 in C. sativus. Independently of the total number of spots, the number of spots whose relative abundance changed significantly as a result of the different $\mathrm{Fe}$ deficiency treatments was within the same range among plant species, with 17,31, and 61 spots for P. dulcis $\times$ P. persica, M. truncatula and B. vulgaris, 57 for C. sativus, and 41 (Li et al., 2008) and 24 (Brumbarova et al., 2008) for S. lycopersicum, respectively. The protein identification rates ranged between 71 and $95 \%$, except for B. vulgaris where only $36 \%$ of the proteins changing in abundance upon Fe limitation were identified, underlying the importance of using plant species with sufficient genomic information for proteomic studies. In A. thaliana root extracts, a high-throughput study of changes induced by Fe limitation was performed using HPLC-MS and iTRAQ (Isobaric Tag for Relative and Absolute Quantification). In this study, 4454 proteins were identified in A. thaliana root extracts and 2882 were reliably quantified; from these, a subset of 101 proteins were identified whose abundance changed upon Fe deficiency (Lan et al., 2011). Finally, a phosphoproteomics study with $A$. thaliana found that among 425 phosphorylated proteins, 45 changed in abundance with Fe deficiency (Lan et al., 2012).

The comparison of proteomes from multiple plant species is not straightforward. This is because of difficulties in handling homology between species, in particular when public genomic data sets are not comprehensive, or in some cases are lacking, as it occurs with B. vulgaris. Furthermore, the reliability of protein identification using database comparisons always has some uncertainty and clearly depends on the stringency of the criteria selected for positive identification, including number of peptides matched and sequence coverage. In addition, because any given proteomic profile is a snapshot of a dynamic process, differences in plant developmental stage and in the manner Fe limitation is imposed can likely lead to final protein profile pictures that may differ significantly. Also, we should keep in mind that changes in spot intensity can be related to post-translational modifications, adding a new layer of complexity to the interpretation of proteomic studies. As a compromise, we used an approach where identified proteins found to change in each plant species were matched to proteins existing in A. thaliana. BLAST searches were run for every protein species found in the different studies and those matches with $E$-values below $\mathrm{e}^{-30}$ were retained as positive Arabidopsis orthologs; if any of the accession numbers overlapped between two or more entries of the same plant species, they were considered redundant and only a single representative entry was retained (Table S1). Also, in an attempt to reduce variability associated with differences in the treatments, the proteomes of $B$. vulgaris and $M$. truncatula plants grown in the absence of $\mathrm{Fe}$, but in the presence of $\mathrm{CaCO}_{3}$, were in principle excluded from the comparison (although data are also provided in Table S1).

Based on this approach, 199 non-redundant Arabidopsis orthologs were found to change in relative abundance with $\mathrm{Fe}$ deficiency: 101, 17, 37, 27, and 32 of them were derived from the Arabidopsis (Lan et al., 2011), M. truncatula, S. lycopersicum, P. dulcis $\times$ P. persica, and C. sativus studies, respectively. These non-redundant root proteins and the results from the BLAST searches are listed in Table S1. Results showing proteins grouped by function (generally as described by authors, although with some modifications) are summarized in Figure 1A and Table S2 (in the latter case with red and green backgrounds indicating proteins increasing or decreasing in relative abundance, respectively). This comparison did not yield a single specific protein species changing in all plant species as a result of Fe limitation, whereas only 15 proteins did show changes in two or more plant species (Figure 1B; Table S2). Among them, six were related to stress defense, three were related to $\mathrm{C}$ metabolism, and three to $\mathrm{N}$ metabolism. The remaining three protein species were a $\beta$-xylosidase involved in cell wall modification, the translation initiation factor ELF5A-1, and the chaperone HSP70-1. In spite of the low number of common protein overlaps, changes in metabolic pathways do follow a similar trend. It should be noted that changes in $\mathrm{Fe}$ homeostasis and transport proteins upon $\mathrm{Fe}$ deficiency were only detected in Arabidopsis using HPLC-MS, because most of these proteins are membrane associated and therefore difficult to solubilize and detect with gel-based 2-DE approaches.

\section{COMMON CHANGES AMONG PLANT SPECIES Oxidative stress and defense}

Oxidative stress was the category containing more specific proteins (five) changing as a result of Fe deficiency in more than one plant species. These changes included increases in superoxide dismutases (CuZnSOD and MnSOD), monodehydroascorbate reductase (MDAR1), peroxidase 12 (PER12) and a decrease in catalase (CAT-2) (Figure 1B; Table S2). These observations point to the strong impact of Fe deficiency on redox homeostasis, not only because free $\mathrm{Fe}$ ions induce reactive oxygen species (ROS) formation via Fenton reactions, but also because many proteins involved in oxidative stress such as peroxidases and catalase are Fe-containing proteins. Some of the changes observed, such as the compensatory mechanism of the SOD isoenzymes in $P$. dulcis $\times P$. persica, M. truncatula and Arabidopsis, as well as the decrease in catalase in S. lycopersicum and $P$. dulcis $\times P$. persica, are likely associated with the decrease in Fe availability. The increases and decreases reported in some of the plant species for the different peroxidase species (i.e., increases in PER12, PER3 and decreases in PER53 and PER21, all of them containing Fe), indicate the complex interplay between $\mathrm{Fe}$ and redox homeostasis mechanisms. Increases in MDAR (in S. lycopersicum and Arabidopsis) and ascorbate peroxidase (APX; in S. lycopersicum) also indicate an induction of the glutathione-ascorbate cycle in response to Fe deficiency.

With regard to defense proteins, only GLP5 (GerminLike-Protein 5) increased in two species, S. lycopersicum 


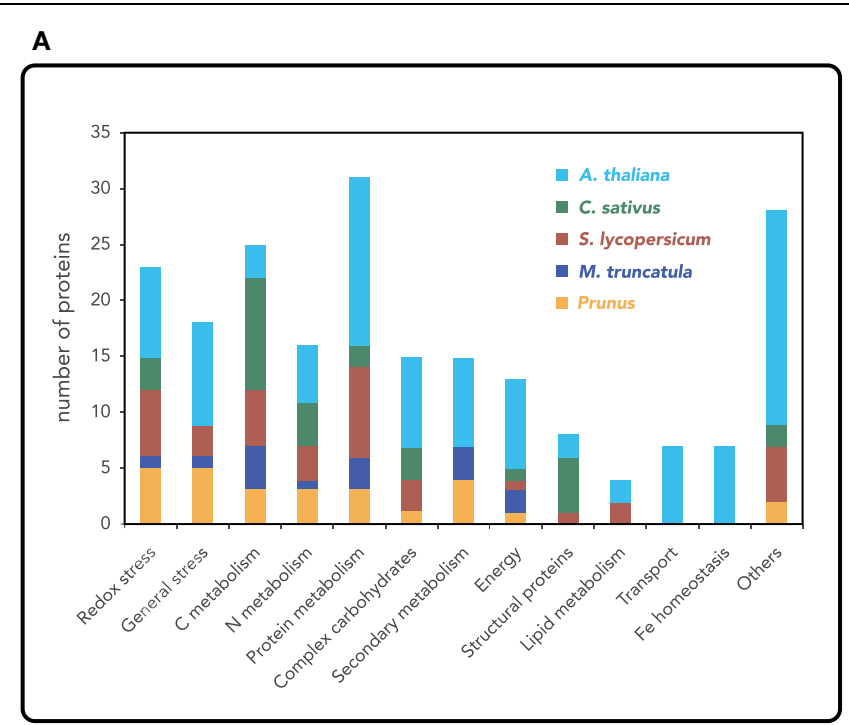

FIGURE 1 | (A) Functional classification of proteins in root extracts that shows changes in relative abundance as a result of Fe deficiency. The number of proteins and functional assignments were obtained from proteomic studies in roots of Arabidopsis thaliana (yellow; Lan et al., 2011), Cucumis sativus (green; Donnini et al., 2010), Solanum lycopersicum (red; Brumbarova et al., 2008; Li et al., 2008), Medicago truncatula (blue; Rodríguez-Celma et al., 2011a) and the Prunus rootstock Prunus dulcis $\times$ Prunus persica (orange; Rodríguez-Celma et al., 2013a). (B) Proteins with changes in relative abundance as a result of Fe deficiency in two or more
B

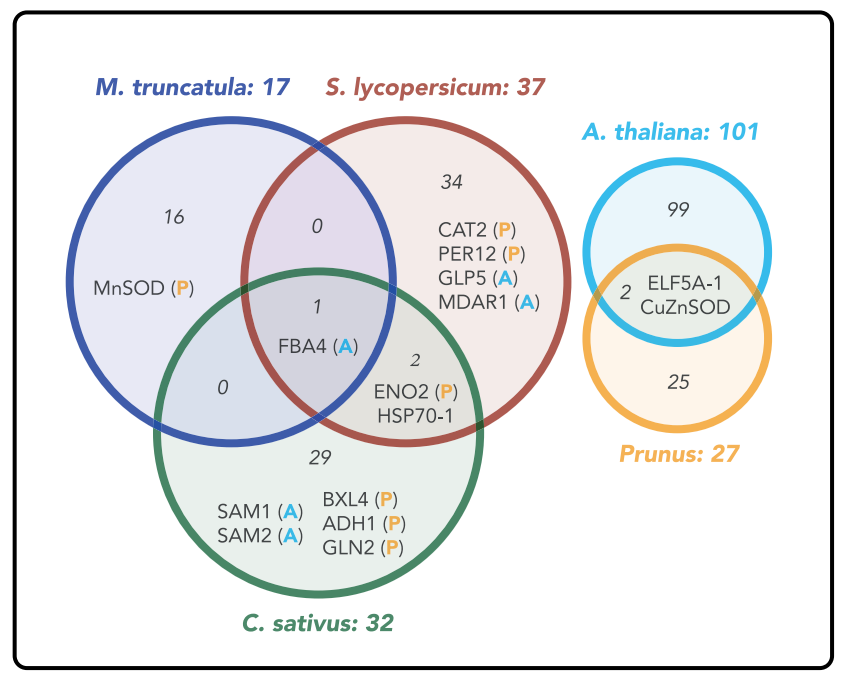

plant species are shown in a Venn diagram. Since the comparison of five species in a single Venn diagram was not fully clear, we used two Venn graphs: in the one in the left, a comparison is made between $C$. sativus, $S$. Iycopersicum, and M. truncatula (letters next to the protein species acronyms indicate whether changes also occur in $A$. thaliana and the Prunus rootstock), whereas the one in the right shows the contrast between $A$. thaliana and the Prunus rootstock. Numbers within the circles indicate the total number of proteins changing in relative abundance as a result of Fe deficiency in each plant species. and Arabidopsis (Figure 1B; Table S2). This protein is plasmodesmata-localized and it is involved in regulating primary root growth by controlling phloem-mediated allocation of resources between the primary and lateral root meristems (Ham et al., 2012). Six glutathione S transferase (GST) proteins changed as a result of Fe deficiency, although no specific overlaps were observed between plant species. The GSTs decreasing in abundance in $P$. dulcis $\times P$. persica (GSTF9 and GSTF4) belong to the phi class, whereas the GSTs increasing in abundance belong to the lambda (GSTL1 in Arabidopsis and GSTL3 in $P$. dulcis $\times P$. persica) and tau (GSTU19 in M. truncatula and GSTU25 in Arabidopsis) classes. The phi class has been linked to tolerance to oxidative stress, whereas the lambda family likely has an oxidoreductase activity and the tau subclass of GST binds fatty acid derivatives (Dixon and Edwards, 2010). Even though the specific functions of these GSTs are not well known, these proteomic data suggest that glutathionylation could be a common detoxifying strategy in Fe-deficient roots.

\section{C metabolism}

Carbon metabolism, and in particular glycolysis, is the metabolic pathway showing the most consistent changes with Fe deficiency at the proteomic level within all plant species studied (also including those grown in the presence of $\mathrm{CaCO}_{3}$ ) (Figure 1B, Table S2). Two or more proteins belonging to this pathway increased in abundance in all plant species, except for Arabidopsis, where only one protein increased, a fructose bisphosphate aldolase for which increases were also found in S. lycopersicum and C. sativus (and also in M. truncatula and B. vulgaris grown with $\mathrm{CaCO}_{3}$ ). Enolase also showed a consistent increase in all species (in M. truncatula in the presence of $\mathrm{CaCO}_{3}$ ) except Arabidopsis. Furthermore, these two proteins were the only ones increasing with Fe deficiency in three of the five plant species studied, highlighting the fact that the metabolic reprogramming of carbon metabolism that occurs upon Fe deficiency is well conserved among species. Increases in the abundance of enzymes from the TCA cycle with Fe deficiency were found in C. sativus and M. truncatula, whereas an increase in PEPC was detected in S. lycopersicum. These proteomic results are in agreement with transcriptomic and biochemical studies that report increases in glycolysis and TCA cycle enzymes upon Fe limitation (see Zocchi, 2006 for a review). Increases in the activity of these pathways could (1) fulfill the increased demand for reducing power and ATP from the up-regulated Fe acquisition mechanisms, including the PM Fe reductase and ATPase, and (2) account for the increases in carboxylates consistently reported in Fe-deficient roots. These holistic proteomic approaches highlight the importance of general metabolic pathways in the elicitation of the stress response mechanisms. In only one case, a protein of the TCA cycle, aconitase in C. sativus, showed a relative decrease in abundance with Fe deficiency. Although aconitase is an Fe containing protein, this decrease has only been reported for $C$. sativus, whereas contrasting changes in aconitase activity as a result of Fe deficiency have been reported in other plant species (Abadía et al., 2002). These apparent inconsistencies with regard to aconitase could be explained by the different ways Fe 
treatments were imposed: in the C. sativus study, seedlings were always grown in the absence of $\mathrm{Fe}$, whereas in the other studies plants were pre-grown with Fe before imposing the Fe-deficiency treatment.

Two enzymes involved in fermentation, alcohol dehydrogenase in $P$. dulcis $\times P$. persica and $C$. sativus and formate dehydrogenase in S. lycopersicum (and in B. vulgaris grown in the presence of $\mathrm{CaCO}_{3}$ ) increased in relative abundance in Fe-deficient roots. These results are also in agreement with previous studies, and have been associated with low intracellular $\mathrm{O}_{2}$ caused by an increased consumption of $\mathrm{O}_{2}$ in Fe-deficient roots (López-Millán et al., 2000, 2009).

\section{N metabolism}

Iron deficiency also has an impact on $\mathrm{N}$ metabolism. Three proteins related to $\mathrm{N}$ metabolism changed in at least two plant species: glutamine synthetase (GLN2) increased in $P$. dulcis $\times$ $P$. persica and C. sativus, S-adenosylmethionine synthase 1 (SAM 1) increased in C. sativus and Arabidopsis, and SAM2 decreased in C. sativus and increased in Arabidopsis (Figure 1B, Table S2). In some species, increases were also observed in enzymes involved in ammonia release [urease (UREG) and dihydrolipoamide dehydrogenase (LPD1) in M. truncatula and an omega amidase in C. sativus], assimilation (GLN1 in Arabidopsis, GLN2 in P. dulcis $\times P$. persica and C. sativus) and amino group transfer (alanine aminotransferase in $C$. sativus and aspartate aminotransferase in M. truncatula grown with $\mathrm{CaCO}_{3}$ ). Overall, these changes indicate an increase in $\mathrm{N}$ recycling that could help to overcome the reduced $\mathrm{N}$ assimilation caused by a decrease in the Fe-containing enzyme nitrite reductase. Decreases in nitrite reductase have been found in M. truncatula and S. lycopersicum at the protein level and in C. sativus at the biochemical level (Borlotti et al., 2012).

Fe deficiency has a conserved impact on SAM metabolism among plant species. Increases in proteins involved in SAM synthesis have been found at the proteomic level in S. lycopersicum, M. truncatula, C. sativus and Arabidopsis. Several authors have already pointed out the central role of SAM in the Fe-deficiency response, not only as a methyl group donor but also as a precursor for the synthesis of nicotianamine, an important Fe chelator, and ethylene, a hormone with a role in the regulation of $\mathrm{Fe}$ acquisition in roots (Waters et al., 2007; Donnini et al., 2010; Lan et al., 2011).

\section{SPECIES- OR TREATMENT-SPECIFIC CHANGES Cell wall}

A significant number of proteins involved in the metabolism of glycosyl compounds are Fe-responsive in all plant species except in M. truncatula (one in P. dulcis $\times$ P. persica, four in S. lycopersicum, three in C. sativus and eight in Arabidopsis), and most of them are localized in the apoplast, suggesting that Fe shortage leads to modifications in cell wall structure and metabolism. Although a more detailed biochemical study is needed to understand these changes, these responses to Fe deficiency seem to be species-specific. In $C$. sativus, decreases were measured for two enzymes involved in the hydrolysis of O-glycosyl compounds (1,4- $\beta$-xylosidase and UDP-glucose dehydrogenase) and an invertase, whereas in $P$. dulcis $\times P$. persica only $1,4-\beta$-xylosidase was found to decrease. In Arabidopsis, in addition to decreases in proteins involved in the transfer of glycosyl groups, lignin catabolism, and hydrolysis, increases were measured in five more proteins: four involved in lignin or cellulose biosynthesis and a $\beta$ glucosidase involved in cellulose degradation. A similar situation was observed in S. lycopersicum, with two cell wall-related proteins increasing and two decreasing. In the studies with C. sativus, the authors explained these changes as a decrease in cell wall biosynthesis that could potentially lead to increased carbon flux toward other metabolic processes such as glycolysis (Donnini et al., 2010); however, the nature of the proteins identified may also reflect hydrolysis of the cell wall as a means to supply carbon skeletons. Irrespective of the specific changes occurring, it can be concluded that cell wall remodeling occurs upon Fe limitation; whereas this may provide carbon for glycolysis or other processes, it is also likely to cause changes in the dynamics of intercellular metabolite trafficking.

\section{Secondary metabolism}

An interesting observation highlighted by our comparison relates to the species-specific changes observed in proteins of secondary metabolism. Even though no overlaps were seen among proteins changing in abundance with Fe deficiency in the different plant species, proteins participating in the riboflavin synthesis pathway increased in M. truncatula (and in B. vulgaris grown in the presence of $\mathrm{CaCO}_{3}$ ), whereas increases in several proteins involved in the phenylpropanoid pathway were observed in Arabidopsis roots and in isoprenoid and flavonoid synthesis in $P$. dulcis $\times$ P. persica. These proteomic results are in agreement with biochemical and transcriptomic data (Rellán-Álvarez et al., 2010; Lan et al., 2011; Rodríguez-Celma et al., 2011b, 2013b) and suggest that Fe deficiency induces the synthesis of different secondary metabolites in roots whose function in the Fe deficiency response deserves further attention.

\section{Energy and ATP-coupled transport processes}

Contrasting results are reported in root proteomes regarding energy-related proteins. In Arabidopsis, significant increases in the abundance of proteins participating in the mitochondrial transport chain, including several subunits of complex I, were observed upon Fe deficiency, whereas in M. truncatula and C. sativus, decreases were found for a subunit of complex I and in subunit $\mathrm{B}$ of the mitochondrial ATPase. Also, the abundance of two vacuolar ATPases was decreased in Fe-deficient Arabidopsis, whereas a higher abundance was reported for a vacuolar ATPase in Fedeficient S. lycopersicum. The decrease in complex I has been confirmed at the biochemical level also in C. sativus (Vigani et al., 2009; Vigani, 2012). These apparent discrepancies could be species-specific or may be explained by the different growing conditions, because Arabidopsis was grown in agar under continuous illumination conditions (including the root) that can alter electron transfer reactions.

\section{Protein metabolism}

It is more difficult to extract general conclusions about changes in protein metabolism related processes induced by Fe deficiency. In Arabidopsis, several translation elongation and initiation factors 
increased in abundance upon Fe limitation, and abundance decreases were also found for some ribosomal components (Lan et al., 2011). In $P$. dulcis $\times P$. persica, an abundance increase in a translation initiation factor was found, but similar changes were not observed in other species. In two species, M. truncatula and $S$. lycopersicum, an increase in proteolysis may take place upon Fe-deficiency based on increases measured in some proteases and in the reorganization of the proteasome machinery. In C. sativus, no changes were measured in proteolytic enzymes, but decreases were observed in structural proteins such as actin and globulins. The authors hypothesized that these proteins could be used as a source of $\mathrm{C}$ and $\mathrm{N}$ during Fe-deficient conditions that cause macronutrient starvation (Donnini et al., 2010).

\section{THE SHOOT PROTEOME}

Changes induced by Fe deficiency in the shoot proteome have only been reported for S. lycopersicum (Herbik et al., 1996). In that study, although apparent differences were observed in the protein profiles of Fe-sufficient and Fe-deficient leaves, protein identification was not pursued because the aim of the work was to identify differences between a mutant $S$. lycopersicum genotype (chloronerva) and wild type.

\section{THE THYLAKOID PROTEOME}

Thylakoid membranes contain the multiprotein photosynthetic complexes photosystems I and II, which include the reaction centers responsible for converting light energy into chemical bond energy, as well as cytochrome $b_{6} / f$ and ATPase complexes. Fe-deficient plants have a reduced number of granal and stromal lamellae per chloroplast and decreased amounts of many thylakoid membrane components including proteins, electron carriers and lipids and this is consistent with marked decreases in photosynthetic rates (Terry, 1980; Terry and Abadía, 1986; Morales et al., 1990).

Changes induced in the thylakoid proteome by Fe-deficiency have been studied in B. vulgaris and Spinacia oleracea using 2-DE blue native (BN) SDS/PAGE in combination with mass spectrometry (Andaluz et al., 2006; Timperio et al., 2007) and by HPLC and tandem mass spectrometry in A. thaliana (Laganowsky et al., 2009). These studies clearly demonstrate that Fe deficiency causes decreases in the relative amounts of electron transfer proteins, including the core and light harvesting components of the PSI and PSII complexes and in cytochrome b6/f, with the largest decreases seen in PSI. The time course studies performed in S. oleracea also indicate that Fe deficiency affects supercomplex organization, by inducing a transient decrease in trimeric and dimeric organization of the light harvesting complex of PSII. These changes might constitute an adaptive strategy to facilitate energy dissipation in Fe-deficient plants (Timperio et al., 2007).

Contrasting results were obtained for ATP synthase, showing no Fe-deficiency induced changes in B. vulgaris and A. thaliana and decreases in $S$. oleracea. Also, the relative amounts of proteins involved in leaf $\mathrm{C}$ fixation, including Rubisco, increased in B. vulgaris, whereas Rubisco decreased significantly in S. oleracea. These results may be species-specific or more likely are related to plant culture conditions, because $S$. oleracea was grown without
Fe from germination, whereas B. vulgaris and Arabidopsis had a preculture period in the presence of Fe. Other changes observed in proteins present in thylakoid preparations upon Fe limitation included increases in enzymes involved in ROS protection, such as ascorbate peroxidase in A. thaliana and SOD in B. vulgaris.

The analyses of exact masses of thylakoid membrane proteins in Arabidopsis also revealed that several proteins undergo posttranslational modifications upon Fe deficiency; for example, the PSII oxygen evolving complex in Fe-deficient Arabidopsis thylakoids was found mostly in its doubly phosphorylated form, while in Fe-sufficient samples the non-phosphorylated form was predominant (Laganowsky et al., 2009).

Results provided in these papers underscore that 2-DE BN SDS/PAGE and HPLC-MS are the techniques more suitable for proteomic studies involving membranes, because they allow the resolution of highly hydrophobic integral membrane proteins. However, the use of other approaches such as 2-DE IEF SDS/PAGE also provides useful complementary information about other sets of proteins tightly linked to membranes.

\section{CONCLUSION AND FUTURE PERSPECTIVES}

As proteomic and transcriptomic techniques have evolved toward high-throughput methodologies such as iTRAQ or the new Illumina technology, it has become more evident that a strict correlation does not exist between observed changes in protein and gene expression profiles. The comparison between changes in proteomic and transcriptomic profiles was out of the scope of this review; however, we have compared the datasets obtained from Arabidopsis Fe-deficient root extracts using iTRAQ and RNA sequencing. This comparison is only possible in Arabidopsis, where the genome is available and both studies have been reported; for other plant species, such as Medicago, the genome is still not finished and there are no reports of iTRAQ profiles. The total number of Arabidopsis transcripts identified was $\sim 26 \mathrm{~K}$, from which 2679 (10\%) were differentially expressed upon Fe deficiency (Rodríguez-Celma et al., 2013b), whereas the total number of proteins identified by iTRAQ was 4454, from which $101(2 \%)$ were differentially accumulated upon Fe deficiency (Lan et al., 2011). Among these, forty-seven protein species (50\% of the differentially accumulated proteins) showed changes in both protein accumulation and gene expression (Table S3). The results of this comparison indicate that both omic approaches are complementary and that the gap in information between them is not only due to methodological limitations, but also reflects the complex regulation of stress responses such as Fe deficiency.

As shown in this review, proteomics has been a powerful tool in the elucidation of general metabolic rearrangements upon $\mathrm{Fe}$ deficiency. Different proteomic approaches have demonstrated that Fe deficiency has a profound impact on $\mathrm{C}$ metabolism and on the arrangement of the photosynthetic machinery, with many of these changes conserved amongst plant species. However, species- and treatment-specific changes have also been revealed that deserve further attention. The full potential of proteomic approaches is quite far from being fully exploited and detailed studies on specific changes unveiled with this holistic technique may yield new insights into the adaptation of plants to Fe deficiency. Future directions for proteomic studies should 
focus on the analysis of sub-proteomes crucial for Fe homeostasis, such as those of plant fluids, isolated organelles, and purified membrane preparations. Recent examples have been published for plasma membrane (Meisrimler et al., 2011; Hopff et al., 2013) and phloem sap (Lattanzio et al., 2013). These studies will help to elucidate the specific roles subcellular compartments may play in Fe homeostasis, and also will allow the discovery of proteins with new functions in these processes, which might remain hidden using other, less targeted approaches.

\section{ACKNOWLEDGMENTS}

The writing of this review was supported in part by the Spanish Ministry of Economy and Competitivity (MINECO; projects AGL2010-16515 and AGL2012-31988, co-financed with FEDER), the Aragón Government (Group A03), and by the US Department of Agriculture, Agricultural Research Service (Agreement number 58-6250-0-008). The contents of this publication do not necessarily reflect the views or policies of the US Department of Agriculture, nor does mention of trade names, commercial products, or organizations imply endorsement by the US Government.

\section{SUPPLEMENTARY MATERIAL}

The Supplementary Material for this article can be found online at: http://www.frontiersin.org/Plant_Nutrition/10.3389/fpls.2013. 00254/abstract

\section{REFERENCES}

Abadía, J., López-Millán, A. F., Rombolà, A., and Abadía, A. (2002). Organic acids and Fe deficiency: a review. Plant Soil 241, 75-86. doi: 10.1023/A:1016093317898

Abadía, J., Vázquez, S., Rellán-Álvarez, R., El-Jendoubi, H., Abadía, A., Álvarez-Fernández, A., et al. (2011). Towards a knowledge-based correction of iron chlorosis. Plant Physiol. Biochem. 49, 471-482. doi: 10.1016/j.plaphy.2011.01.026

Andaluz, S., López-Millán, A. F., De las Rivas, J., Aro, E. M., Abadía, J., and Abadía, A. (2006). Proteomic profiles of thylakoid membranes and changes in response to iron deficiency. Photosynth. Res. 89, 141-155. doi: 10.1007/s11120006-9092-6

Borlotti, A., Vigani, G., and Zocchi, G. (2012). Iron deficiency affects nitrogen metabolism in cucumber (Cucumis sativus L.) plants. BMC Plant Biol. 12:189. doi: 10.1186/1471-2229-12-189

Brumbarova, T., Matros, A., Mock, H. P., and Bauer, P. (2008). A proteomic study showing differential regulation of stress, redox regulation and peroxidase proteins by iron supply and the transcription factor FER. Plant J. 54, 321-334. doi: 10.1111/j.1365313X.2008.03421.x
Dixon, D. P., and Edwards, R. (2010). Glutathione Transferases. Arabidopsis Book 8:e0131. doi: 10.1199/tab.0131

Donnini, S., Prinsi, B., Negri, A. S., Vigani, G., Espen, L., and Zocchi, G. (2010). Proteomic characterization of iron deficiency responses in Cucumis sativus L. roots. BMC Plant Biol. 10:268. doi: 10.1186/14712229-10-268

Ham, B. K., Li, G., Kang, B. H., Zeng, F., and Lucas, W. J. (2012). Overexpression of Arabidopsis plasmodesmata germin-like proteins disrupts root growth and development. Plant Cell 24, 3630-3648. doi: 10.1105/tpc. 112.101063

Herbik, A., Giritch, A., Horstmann, C., Becker, R., Balzer, H. J., Baumlein, H., et al. (1996). Iron and copper nutrition-dependent changes in protein expression in a tomato wild type and the nicotianamine-free mutant chloronerva. Plant Physiol. 111, 533-540. doi: 10.1104/pp.111. 2.533

Hindt, M. N., and Guerinot, M. L. (2012). Getting a sense for signals: regulation of the plant iron deficiency response. Biochim. Biophys. Acta 1823, 1521-1530. doi: 10.1016/j.bbamcr.2012.03.010

Hopff, D., Wienkoop, S., and Lüthje, S. (2013). The plasma membrane proteome of maize roots

Table S1 | Tables of BLAST results from proteins identified in roots from Prunus dulcis x Prunus persica (Rodríguez-Celma et al., 2013a), Medicago truncatula (Rodríguez-Celma et al., 2011a), Beta vulgaris (Rellán-Álvarez et al., 2010), Cucumis sativus (Donnini et al., 2010), and Solanum

Iycopersicum (Herbik et al., 1996; Brumbarova et al., 2008; Li et al., 2008). Spot nomenclature in the first column is that used in the original papers. BLAST searches were performed in the NCBI website (http://blast.ncbi. nlm.nih.gov/Blast.cgi) against the Arabidopsis database (taxid:3701) in March 2013. BLAST annotations were assigned as not hit when BLAST E-values were higher than $1 \mathrm{e}^{-30}$. If any of the accession numbers overlapped between two or more entries of the same plant species, they were considered redundant and only a single representative entry was retained.

Table S2 | Comparison of changes observed upon Fe deficiency in the non-redundant Arabidopsis root proteomes obtained from Arabidopsis thaliana (Lan et al., 2011) and from BLAST results in Cucumis sativus, Solanum lycopersicum, Medicago truncatula and Prunus dulcis $\times$ Prunus persica. Red and green backgrounds indicate increases and decreases, respectively, in protein abundance upon Fe deficiency. A blue background in the first column marks proteins showing changes in two or more plant species (not considering the $M$. truncatula and $B$. vulgaris treatments including $\mathrm{CaCO}_{3}$, which are included in the Table in the last two columns in white characters).

Table S3 | List of Aradidopsis thaliana gene identifiers showing changes in both protein accumulation (Lan et al., 2011) and gene expression (Rodríguez-Celma et al., 2013b).

grown under low and high iron conditions. J. Proteomics doi: 10.1016/j.jprot.2013.01.006. [Epub ahead of print].

Laganowsky, A., Gomez, S. M. Whitelegge, J. P., and Nishio, J. N. (2009). Hydroponics on a chip: analysis of the $\mathrm{Fe}$ deficient Arabidopsis thylakoid membrane proteome. J. Proteomics 72, 397-415. doi: 10.1016/j.jprot.2009. 01.024

Lan, P., Li, W., Wen, T. N., and Schmidt, W. (2012). Quantitative phosphoproteome profiling of iron-deficient Arabidopsis roots. Plant Physiol. 159, 403-417. doi: 10.1104/pp.112.193987

Lan, P., Li, W., Wen, T. N., Shiau, J. Y., Wu, Y. C., Lin, W., et al. (2011). iTRAQ protein profile analysis of Arabidopsis roots reveals new aspects critical for iron homeostasis. Plant Physiol. 155, 821-834. doi: 10.1104/pp.110.169508

Lattanzio, G., Andaluz, S., Matros, A., Calvete, J. J., Kehr, J., Abadía, A., et al. (2013). Protein profile of Lupinus texensis phloem sap exudates: searching for $\mathrm{Fe}$ and $\mathrm{Zn}$ containing proteins. Proteomics doi: 10.1002/pmic.201200515. [Epub ahead of print].

Li, J., Wu, X. D., Hao, S. T., Wang, X. J., and Ling, H. Q. (2008). Proteomic response to iron deficiency in tomato root Proteomics 8, 2299-2311. doi: 10.1002/pmic. 200700942

López-Millán, A. F., Morales, F., Andaluz, S., Gogorcena, Y., Abadía A., De Las Rivas, J., et al. (2000). Responses of sugar beet roots to iron deficiency. Changes in carbon assimilation and oxygen use. Plant Physiol. 124, 885-898. doi: 10.1104/pp.124.2.885

López-Millán, A. F., Morales, F., Gogorcena, Y., Abadía, A., and Abadía, J. (2009). Metabolic responses in iron deficient tomato plants. J. Plant Physiol. 166, 375-384. doi: 10.1016/j.jplph.2008.06.011

McLean, E., Cogswell, M., Egli, I., Wojdyla, D., and de Benoist, B. (2009). Worldwide prevalence of anaemia, WHO vitamin and mineral nutrition information system, 1993-2005. Public Health Nutr. 12, 444-454. doi: 10.1017/S1368980008002401

Meisrimler, C. N., Planchon, S., Renaut, J., Sergeant, K., and Lüthje, S. (2011). Alteration of plasma membrane-bound redox systems of iron deficient pea roots by chitosan. J. Proteomics 74, 1437-1449. doi: 10.1016/j.jprot.2011.01.012

Morales, F., Abadía, A., and Abadía, J. (1990). Characterization of the xanthophyll cycle and other 
photosynthetic pigment changes induced by iron deficiency in sugar beet (Beta vulgaris L.). Plant Physiol. 94, 607-613. doi: 10.1104/ pp.94.2.607

Morgan, J. W., and Anders, E. (1980). Chemical composition of Earth, Venus, and Mercury. Proc. Natl. Acad. Sci. U.S.A. 77, 6973-6977. doi: 10.1073/pnas.77.12.6973

Palmer, C. M., and Guerinot, M. L. (2009). Facing the challenges of $\mathrm{Cu}$, $\mathrm{Fe}$ and $\mathrm{Zn}$ homeostasis in plants. Nat. Chem. Biol. 5, 333-340. doi: 10.1038/nchembio. 166

Rellán-Álvarez, R., Andaluz, S., Rodríguez-Celma, J., Wohlgemuth, G., Zocchi, G., Álvarez-Fernández, A., et al. (2010). Changes in the proteomic and metabolic profiles of Beta vulgaris root tips in response to iron deficiency and resupply. BMC Plant Biol. 10:120. doi: 10.1186/1471-2229-10-120

Rodríguez-Celma, J., Lattanzio, G., Grusak, M. A., Abadía, A., Abadía, J., and López-Millán, A. F. (2011a). Root responses of Medicago truncatula plants grown in two different iron deficiency conditions: changes in root protein profile and riboflavin biosynthesis. J. Proteome Res. 10, 2590-2601. doi: $10.1021 /$ pr2000623

Rodríguez-Celma, J., Vázquez-Reina, S., Orduna, J., Abadía, A., Abadía, J., Álvarez-Fernández, A., et al. (2011b). Characterization of flavins in roots of Fe-deficient strategy I plants, with a focus on Medicago truncatula. Plant Cell Physiol. 52, 2173-2189. doi: 10.1093/pcp/pcr149

Rodríguez-Celma, J., Lattanzio, G., Jiménez, S., Briat, J. F., Abadía, J., Abadía, A., et al. (2013a). Changes induced by $\mathrm{Fe}$ deficiency and Fe resupply in the root protein profile of a peach-almond hybrid rootstock. J. Proteome Res. 12, 1162-1172. doi: 10.1021/pr300763c Rodríguez-Celma, J., Lin, W.-D., Fu, G.-M., Abadía, J., López-Míllán, A.-F., and Schmidt, W. (2013b). Mutually exclusive alterations in secondary metabolism are critical for the uptake of insoluble iron compounds by Arabidopsis and Medicago truncatula. Plant Physiol. 162, 1473-1485. doi: 10.1104/pp.113.220426

Römheld, V., and Marschner, H. (1983). Mechanism of iron uptake by peanut plants: I. Fe reduction, chelate splitting, and release of phenolics. Plant Physiol. 71, 949-954. doi: 10.1104/ pp.71.4.949

Schmidt, W., and Buckhout, T. J. (2011). A hitchhiker's guide to the Arabidopsis ferrome. Plant Physiol. Biochem. 49, 462-470. doi: 10.1016/j.plaphy. 2010.12.001
Terry, N. (1980). Limiting factors in photosynthesis. I. Use of iron stress to control photochemical capacity in vivo. Plant Physiol. 65, 114-120. doi: 10.1104/pp.65.1.114

Terry, N., and Abadía, J. (1986) Function of iron in chloroplast. J. Plant Nutr. 9, 609-646. doi: 10.1080/01904168609363470

Timperio, A. M., D’Amici, G. M., Barta, C., Loreto, F., and Zolla, L. (2007). Proteomics, pigment composition, and organization of thylakoid membranes in iron-deficient spinach leaves. J. Exp. Bot. 58, 3695-3710.

Vigani, G. (2012). Discovering the role of mitochondria in the iron deficiency-induced metabolic responses of plants. J. Plant Physiol. 169, 1-11. doi: 10.1016/j.jplph.2011.09.008

Vigani, G., Maffi, D., and Zocchi, G. (2009). Iron availability affects the function of mitochondria in cucumber roots. New Phytol. 182, 127-136. doi: 10.1111/j.1469-8137.2008.02747.x

Waters, B. M., Lucena, C., Romera F. J., Jester, G. G., Wynn, A. N., Rojas, C. L., et al. (2007). Ethylene involvement in the regulation of the $\mathrm{H}(+)$-ATPase CsHAl gene and of the new isolated ferric reductase CsFRO1 and iron transporter CsIRT1 genes in cucumber plants. Plant Physiol. Biochem. 45, 293-301. doi: 10.1016/j.plaphy.2007.03.011
Zocchi, G. (2006). "Metabolic changes in iron-stressed dicotyledoneus plants," in Iron Nutrition in Plants and Rhizospheric Microorganisms, eds L. L. Barton and J., Abadía (Dordrecht: Springer), 359-370.

Conflict of Interest Statement: The authors declare that the research was conducted in the absence of any commercial or financial relationships that could be construed as a potential conflict of interest.

Received: 24 April 2013; accepted: 23 June 2013; published online: 25 July 2013.

Citation: López-Millán A-F, Grusak MA, Abadía $A$ and Abadia J (2013) Iron deficiency in plants: an insight from proteomic approaches. Front. Plant Sci. 4:254. doi: 10.3389/fpls.2013.00254

This article was submitted to Frontiers in Plant Nutrition, a specialty of Frontiers in Plant Science.

Copyright (c) 2013 López-Millán, Grusak, Abadia and Abadia. This is an open-access article distributed under the terms of the Creative Commons Attribution License, which permits use, distribution and reproduction in other forums, provided the original authors and source are credited and subject to any copyright notices concerning any third-party graphics etc. 